\title{
Bibliothèques de collèges à Paris au Moyen-Âge : quand les réguliers inspirent les séculiers
}

\section{Marion Bernard-Schweitzer}

\section{(2) OpenEdition}

1 Journals

Édition électronique

URL : http://journals.openedition.org/rbnu/869

DOI : $10.4000 /$ rbnu.869

ISSN : 2679-6104

Éditeur

Bibliothèque nationale et universitaire de Strasbourg

Édition imprimée

Date de publication : 1 mai 2017

Pagination : 40-47

ISBN : 9782859230661

ISSN : 2109-2761

Référence électronique

Marion Bernard-Schweitzer, « Bibliothèques de collèges à Paris au Moyen-Âge : quand les réguliers inspirent les séculiers », La Revue de la BNU [En ligne], 15 | 2017, mis en ligne le 01 novembre 2019, consulté le 10 décembre 2020. URL : http://journals.openedition.org/rbnu/869 ; DOI : https://doi.org/ $10.4000 /$ rbnu. 869

\section{(c) (1) (ㅇ)}

La Revue de la BNU est mise à disposition selon les termes de la Licence Creative Commons Attribution - Pas d'Utilisation Commerciale - Partage dans les Mêmes Conditions 4.0 International. 

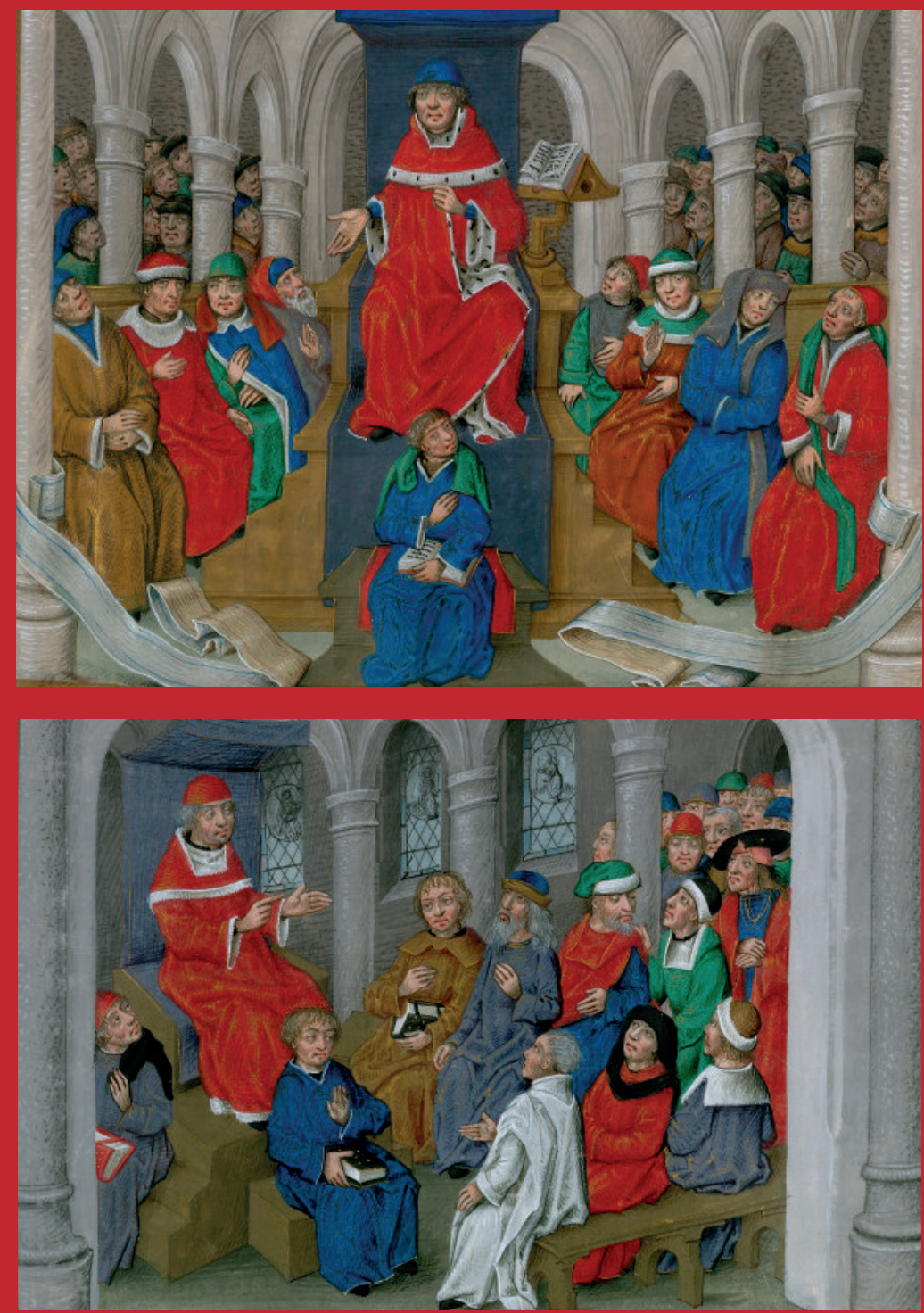

Miniatures représentant Jean Gerson enseignant, in Jean Gerson, Thomas a Kempis, Recueil de spiritualité, vers 1480 (coll. Bibliothèque municipale de Valenciennes)

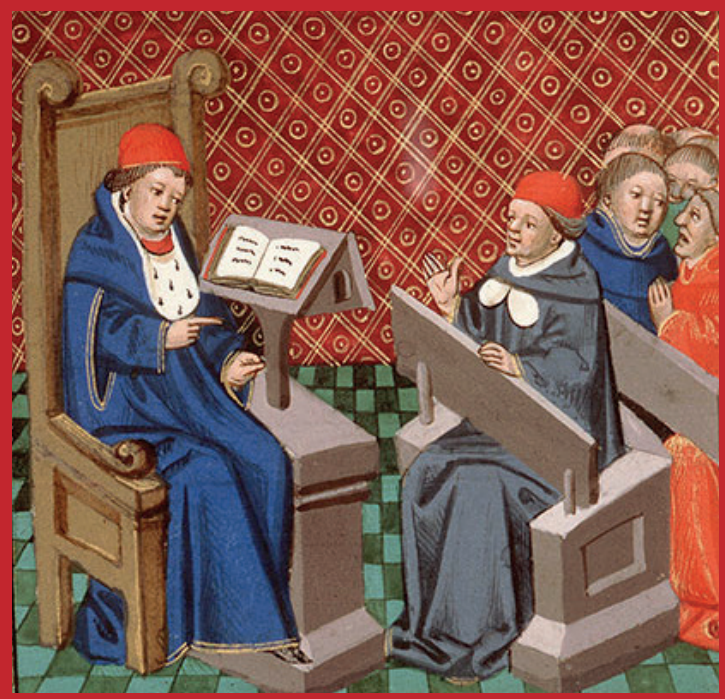

Miniature représentant l'enseignement de la médecine, in Bartholomaeus Anglicus, Livre des propriétés des choses, 1447 (coll. Bibliothèque municipale d'Amiens) 


\section{BIBLIOTHÈQUES DE COLLÈGES À PARIS AU MOYEN ÂGE : quand les réguliers inspirent les séculiers}

PAR MARION BERNARD-SCHWEITZER

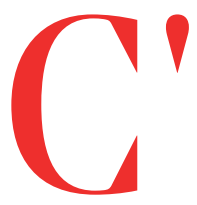

lèges séculiers font leur apparition, principalement sur la rive gauche de la Seine ; le mouvement atteint son apogée au $14^{\mathrm{e}}$ siècle. Ces établissements séculiers sont destinés à héberger les étudiants les moins favorisés de l'Université de Paris, en leur offrant le gîte et le couvert. Jusqu'alors, en effet, les étudiants démunis et qui n'étaient pas originaires de la capitale se voyaient contraints de subvenir à leurs frais à leur logement une chambre chez l'habitant ou chez le maître dont ils suivaient les cours - ; à ces frais venaient s'ajouter tous ceux occasionnés par la poursuite d'études supérieures ${ }^{1}$, qui ne leur permettaient pas de s'y consacrer entièrement, voire les contraignaient à y renoncer au bout de quelque temps. La condition de pauvreté est donc régulièrement mentionnée, dans les statuts des collèges, comme nécessaire pour accéder à une bourse ; la générosité des fondateurs n'est cependant pas octroyée sans contrepartie. Le prélat Robert de Sorbon, fondateur en 1257 du collège de Sorbonne, est le premier à faire mention de progrès à réaliser par les boursiers de son collège ; assiduité, progrès, obtention de grades universitaires sont autant de conditions exigées des étudiants pour conserver leur bourse.

D'une vocation d'abord hospitalière, les collèges vont peu à peu - et en cela la fondation du collège de Sorbonne a été décisive - évoluer vers une dimension plus proprement intellectuelle ; et c'est dans ce cadre qu'ils vont proposer à leurs boursiers l'accès à une bibliothèque d'étude, mettant ainsi à leur disposition des instruments de travail particulièrement coûteux et peu facilement accessibles à un simple étudiant ${ }^{2}$. Quelle a été, sur ce point, l'influence des bibliothèques monastiques préexistantes?

\section{des collèges séculiers}

C'est dans la seconde moitié du $13^{e}$ siècle que certains collèges séculiers commencent à se doter d'une bibliothèque; on peut y voir l'influence des couvents réguliers, notamment des ordres mendiants. Depuis leur fondation au début du $13^{\mathrm{e}}$ siècle, franciscains et 
dominicains se sont en effet solidement implantés dans les grandes villes; dans les centres universitaires, afin de permettre à leurs membres de fréquenter les universités, ils ont établi des maisons d'études, ou "studia ", dotées d'importantes bibliothèques ${ }^{3}$ - ainsi, à Paris, le couvent des dominicains rue Saint-Jacques. La place que prennent bientôt les maîtres issus des ordres mendiants au sein de la faculté de théologie de l'Université de Paris ne manque pas de provoquer des conflits avec les maîtres séculiers ; la cause en est l'efficacité de l'enseignement des frères mendiants qui forment de très habiles prédicateurs, au rayonnement intellectuel éclipsant les séculiers. Cette formation s'appuie en particulier sur les ouvrages et autres instruments de travail mis à la disposition des étudiants mendiants au sein des «studia » de leur ordre ${ }^{4}$.

C'est dans ce contexte qu'intervient en 1257 la fondation du collège séculier de Sorbonne, qui se démarque des fondations précédentes en accueillant non plus de jeunes étudiants débutant leurs études à la faculté des arts, mais des maîtres ès arts commençant leurs études de théologie ${ }^{5}$. Cet élément, ainsi que la présence, dès l'origine, d'une bibliothèque ${ }^{6}$, destinée à accompagner les boursiers dans de brillantes études en théologie, montre la manière dont Robert de Sorbon, et par la suite d'autres fondateurs de collèges séculiers se sont inspirés des couvents mendiants, afin d'œuvrer au renouveau du clergé séculier, trop éclipsé, selon certains, par les ordres mendiants. Ainsi une étude approfondie de deux collections léguées au collège de Sorbonne dans les années 1270, par Robert de Sorbon puis Gérard d'Abbeville - théologien actif dans la polémique contre les ordres mendiants - montre que le contenu de cette première bibliothèque ne différait pas fondamentalement de celui d'une bibliothèque de "studium » mendiant : on y trouvait un grand nombre de manuscrits relevant de l'exégèse et de la pastorale, en conformité avec la volonté de Robert de Sorbon, le fondateur, de former dans ce collège des théologiens habiles dans l'art de prêcher ${ }^{7}$.

Parmi les manuscrits qui forment le noyau de la bibliothèque du collège de Sorbonne, on trouve notamment des ouvrages d'un genre nouveau, apparu au $13^{\mathrm{e}}$ siècle en appui au travail universitaire et à l'activité de prédication des ordres mendiants ${ }^{8}$. Ce sont des concordances des mots de la Bible, ou encore des tables alphabétiques des matières - plusieurs de ces instruments, concordance alphabétique de la Bible, index alphabétique des œuvres des Pères ou de saint Augustin, se retrouvent d'ailleurs dans la bibliothèque de Gérard d'Abbeville ${ }^{9}$, dont il fit don au collège de Sorbonne. Apparus chez les cisterciens, développés par les ordres mendiants pour le besoin de la prédication, ces instruments de travail furent rapidement et fortement utilisés dans les centres universitaires, dont Paris, où ils servirent d'appui au travail intellectuel.

Si le collège de Sorbonne fut le premier à se doter dès l'origine d'une bibliothèque, il fut par la suite imité par la plupart des fondations séculières ; c'est ainsi que se constituèrent au sein des collèges séculiers de véritables bibliothèques d'étude pour l'usage des boursiers - dans le cas du collège de Sorbonne, la bibliothèque était même ouverte à l'ensemble de l'Université de Paris. Rapidement, des méthodes de gestion de ces fonds sont établies, sous l'influence, sans doute, des bibliothèques monastiques.

\section{L'influence des bibliothèques monastiques sur l'organisation des bibliothèques de collèges}

Les abbayes des ordres monastiques possédaient en effet, elles aussi, leurs propres bibliothèques; les collèges universitaires séculiers ont donc pu s'inspirer des pratiques de gestion des collections déjà établies dans ces monastères. Ainsi l'abbaye des chanoines réguliers de Saint-Victor de Paris, l'un des grands centres intellectuels du $12^{\mathrm{e}}$ siècle, comportait une importante bibliothèque. Un document composé avant 1139, le Liber ordinis Sancti Victoris Parisiensis, fait déjà état de deux fonds d'ouvrages distincts : les « libri communes ", qui sont les ouvrages laissés en libre accès par le bibliothécaire, l'« armarius »; et les « libri minores et non cotidiani ", qui pouvaient être prêtés aux chanoines ${ }^{10}$. Cette répartition des collections en deux fonds d'usage distinct est ensuite reprise dans plusieurs documents, et notamment par l'ordre des frères prêcheurs : ainsi Humbert de Romans, dans ses législations rédigées pour l'ordre dominicain, fait référence à une bibliothèque d'usuels enchaînés sur des pupitres pour le libre accès ${ }^{11}$. 


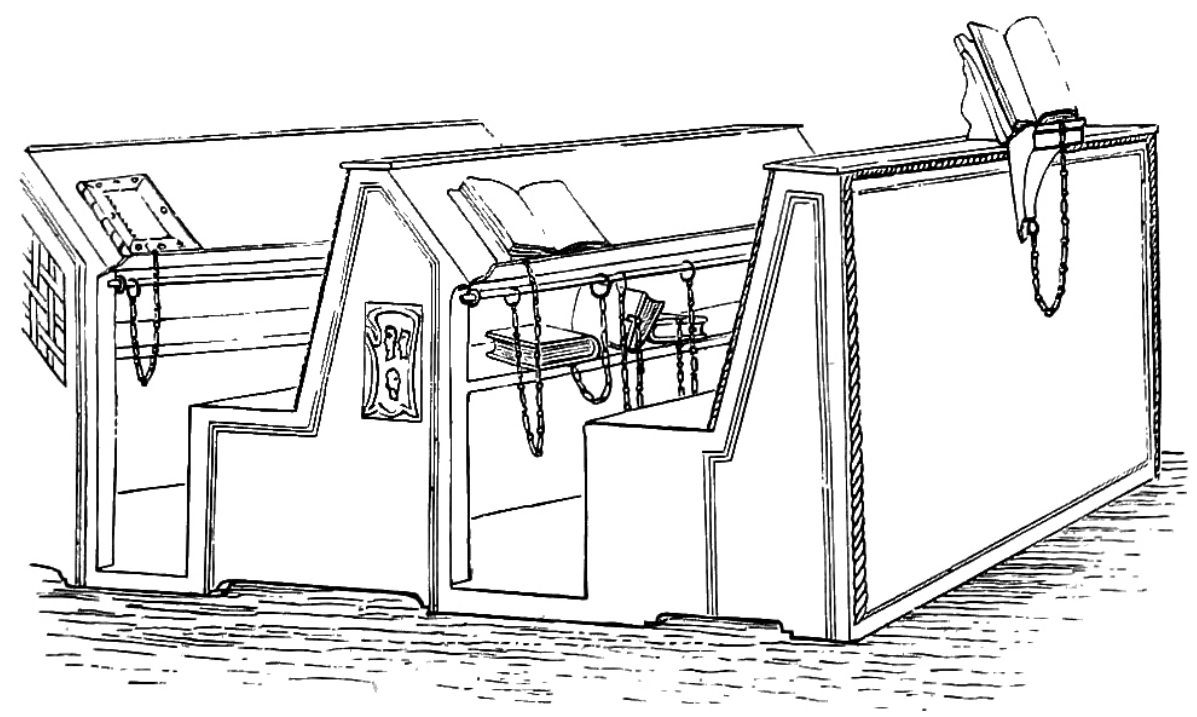

Pupitres de la bibliothèque Malatestiana de Césène, en Italie (in J. W. Clark, Libraries in the medieval and renaissance periods, Cambridge, 1894). C'est ainsi que devaient se présenter les manuscrits enchaînés, en libre accès, dans les bibliothèques de collèges parisiens.

Le collège de Sorbonne va reprendre ce mode de classement à la fin du $13^{\mathrm{e}}$ siècle, afin de réorganiser l'important fonds de manuscrits dont il est devenu le détenteur - plus de mille volumes, ce qui en fait une bibliothèque de premier ordre. Lorsque le proviseur Pierre de Villepreux (1286-1304) prend la décision de réorganiser la bibliothèque ${ }^{12}$, le principe retenu est celui de la constitution de deux fonds : les ouvrages les plus demandés et les plus utiles pour les boursiers sont enchaînés, selon un classement reprenant les disciplines enseignées à l'Université de Paris, sur des pupitres au sein de la "libraria communis ", afin qu'ils soient utiles au plus grand nombre : « quia bonum commune divinius est quam bonum unius ${ }^{13}$. Au début du $14^{\text {e }}$ siècle, le terme de "libraria communis » est remplacé par celui de " libraria magna " (grande bibliothèque) - par opposition à la «libraria parva» (petite bibliothèque), qui contenait les ouvrages moins usuels, les « libri minores ». Ce principe d'organisation des fonds pourrait même être contenu en germe dans le testament (1271) de Gérard d'Abbeville, au sein $\mathrm{du}$ passage relatif au legs effectué à la bibliothèque du collège de Sorbonne ; il y établit diverses clauses ${ }^{14}$ dont la dernière concerne la conservation des ouvrages, dont certains devraient être enchaînés ${ }^{15}$.
Quoi qu'il en soit, à compter de l'adoption en 1289 par la bibliothèque du collège de Sorbonne de ce mode de classement, celui-ci fut adopté par la majorité des bibliothèques religieuses de l'époque : une salle, la " libraria magna ", où se trouvaient, enchaînés sur des pupitres, les ouvrages considérés comme usuels; et une seconde collection, la « libraria parva ", où les ouvrages étaient conservés - souvent dans des coffres - et pouvaient être empruntés. Soulignons que la « libraria parva», malgré son nom, pouvait être plus importante, en volumétrie, que la « libraria magna ».

\section{Deux exemples : les bibliothèques des collèges du Trésorier et de Maître-Gervais}

Deux collèges séculiers parisiens peuvent plus particulièrement illustrer ce propos sur les bibliothèques de collèges : ce sont ceux du Trésorier et de MaîtreGervais. Fondés tous deux par de pieux bienfaiteurs d'origine normande, ils possèdent une bibliothèque qui, quoique sans aucune commune mesure avec les très riches collections des collèges de Sorbonne et de Navarre, est relativement bien connue. 


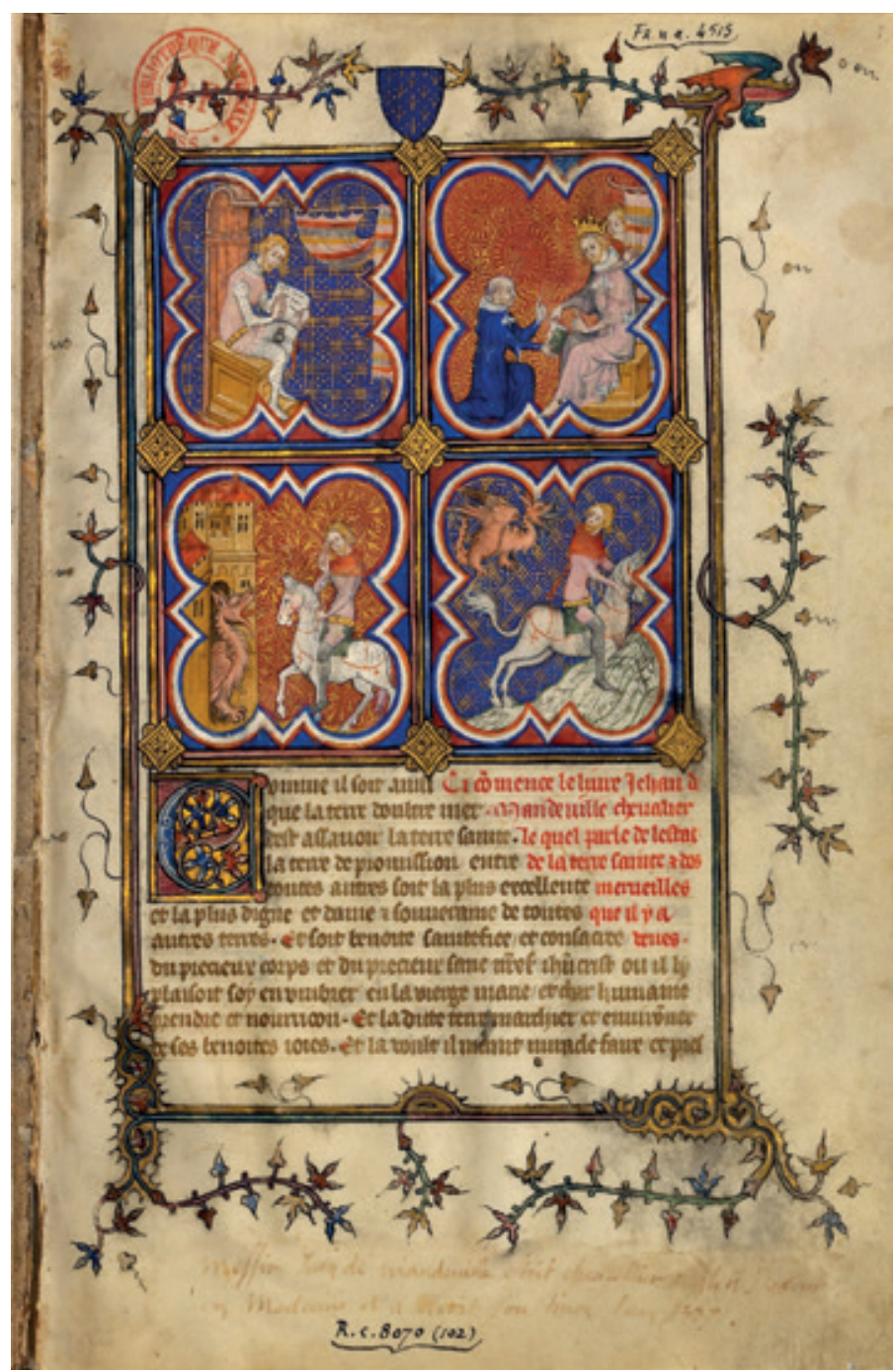

Incipit du Livre des merveilles $d u$ monde, de Jean de Mandeville. Médaillon en haut à droite : Gervais Chrétien offrant l'ouvrage à Charles V, fondateur du collège de Maître-Gervais (coll. Bibliothèque nationale de France).

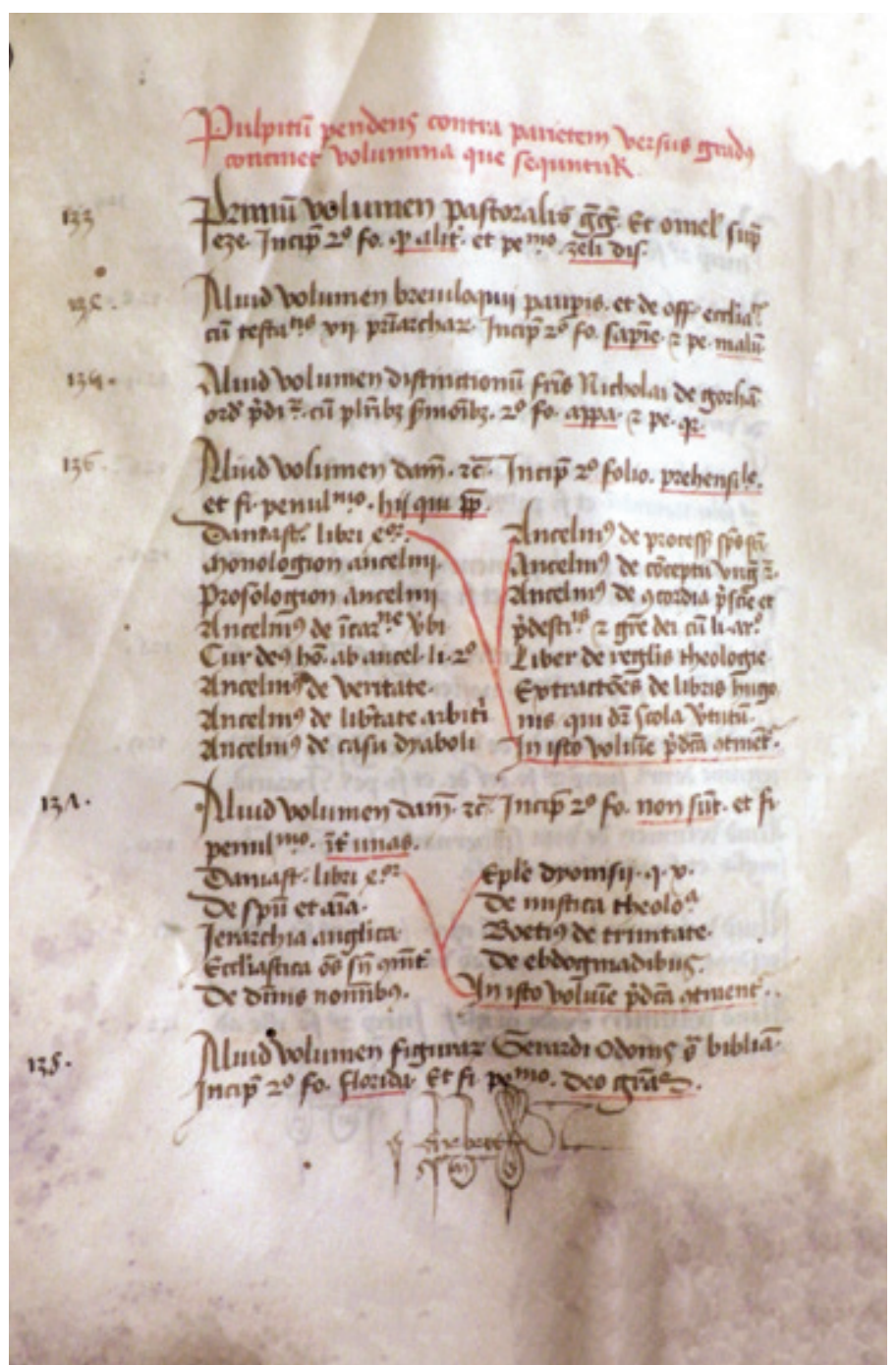

Inventaire de la bibliothèque du collège du Trésorier, 1437 (p. 19 ; coll. Archives nationales) 
Le collège du Trésorier est fondé à Paris en novembre 1268 par Guillaume de Sâane, trésorier de l'église de Rouen. Il est situé rue de la Harpe et destiné à accueillir vingt-quatre étudiants originaires du diocèse de Rouen : douze en arts et douze en théologie. Outre la date de sa fondation assez proche de celle du collège de Sorbonne, il présente plusieurs autres similitudes avec ce dernier : l'accueil d'étudiants en théologie est là aussi prévu dès l'origine, de même que la présence d'une bibliothèque, mentionnée dans les statuts de $1280^{16}$. Si la rareté des sources entrave notre connaissance $\mathrm{du}$ collège $\mathrm{du}$ Trésorier pour la fin $\mathrm{du} 13^{\mathrm{e}}$ siècle et la majeure partie du $14^{\mathrm{e}}$, il est cependant mieux connu au $15^{\mathrm{e}}$ siècle, entre autres grâce à un inventaire de ses biens, daté de $1437^{17}$. Celui-ci comprend notamment une description des collections de sa bibliothèque, faisant de lui l'un des rares collèges parisiens dont la bibliothèque médiévale peut être reconstituée ${ }^{18}$.

L'inventaire des livres

de la bibliothèque en libre

accès, ou " magna libraria », a

été réalisé en suivant l'ordre des

pupitres sur lesquels ils étaient en-

chaînés : on compte ainsi 145 volumes, répartis sur trois pupitres simples - avec une moyenne d'environ dix volumes par pupitre - et six pupitres doubles - avec une moyenne d'environ quinze à vingt volumes par pupitre ${ }^{19}$. Les 147 volumes de la " parva libraria », quant à eux, sont conservés dans de grandes armoires, dans la chapelle. On voit bien ici la reprise de la distinction entre grande et petite bibliothèque, popularisée par le collège de Sorbonne ; et comme dans la fondation de Robert de Sorbon, le fonds est principalement constitué d'ouvrages relevant du champ de la théologie - 227 volumes de théologie sur les 299 mentionnés par l'inventaire ${ }^{20}$.

Le collège de Maître-Gervais, quant à lui, a été fondé en 1371, soit plus d'un siècle après ceux de Sorbonne et du Trésorier. Son fondateur, Gervais Chrétien, médecin du roi Charles V, supervise en détail sa fondation et préside à la rédaction, en $1378^{21}$, de ses statuts.
Il semble que, là encore, l'existence de la bibliothèque soit, à quelques années près, aussi ancienne que l'établissement lui-même : le fonctionnement en est fixé par le chapitre huit des statuts de 1378. Une distinction entre deux fonds est réalisée (" libraria artistarum " et " libraria theologorum »), mais on ne retrouve pas les mêmes logiques qu'à la bibliothèque du collège de Sorbonne. La description, faite par Gervais Chrétien, des deux bibliothèques ne semble correspondre tout d'abord qu'à une question pratique : chaque aile du bâtiment, l'une destinée aux boursiers en théologie, droit ou médecine, l'autre aux boursiers en arts, aura sa bibliothèque « ita quod in qualibet duarum domorum erit una libraria ${ }^{22}$, de sorte qu'il y ait dans chacun des bâtiments un espace dédié à la lecture et à la consultation des livres. Une seconde distinction est ensuite introduite par le fondateur : les fonds seront en effet bien différenciés en fonction du public auquel ils sont destinés, " artiens » ou étudiants des facultés supérieures : " ita quod in libraria theologorum erunt libri ad theologos et medicos pertinentes, et libri logicales et philosophiae et mathematicales in libraria artistarum $»{ }^{23}$. Chacune des deux bibliothèques semble donc avoir un fonds, un public et une destination bien précis ; cependant tous les livres y sont enchaînés, sans distinction (« cum cathenis ligabuntur libri ») ${ }^{24}$, jusqu'aux livres de droit légués par un bienfaiteur juriste, qui doivent être conservés enchaînés dans la chambre de l'étudiant canoniste du collège ${ }^{25}$. En 1414, le testament d'un autre bienfaiteur, Guillaume Vauchis, fait encore état de cette bipartition du fonds et de l'enchaînement des ouvrages : l'ensemble des manuscrits constituant le legs est divisé en deux parties, la première pour la " libraria theologorum collegii magistri Gervasii Christiani », et la seconde pour la « libraria artistarum ». Et le donateur précise bien « quod omnes incathenentur in libraria predicta infra octo dies ab eorum tradicione $"{ }^{26}$. Telle qu'elle est décrite dans les statuts, et qu'elle apparaît par la 
suite dans les sources, la double bibliothèque du collège de Maître-Gervais semble donc être un lieu d'étude, destiné à la consultation sur place des ouvrages, et non une bibliothèque de prêt.

Le fondateur détaille dans les statuts le fonctionnement de la bibliothèque, selon des conditions que l'on retrouve en usage dans nombre de collèges. Ainsi, son usage est théoriquement réservé aux seuls boursiers ${ }^{27}$, à quelques exceptions près. Seuls quelques boursiers de confiance peuvent se voir confier les clés de l'une ou l'autre bibliothèque. Il est permis aux boursiers d'y introduire un étudiant extérieur à l'établissement, avec la permission d'un officier du collège, et à condition de ne pas le laisser seul dans la pièce et de le surveiller ${ }^{28}$. En cas de vol d'un ou plusieurs ouvrages, le boursier qui s'est porté caution est donc considéré comme responsable et chargé de remédier au préjudice subi. Le témoignage, au $15^{\mathrm{e}}$ siècle, de l'astrologue Simon de Phares, qui a eu accès à la bibliothèque du collège et y a consulté des ouvrages, corrobore cet aspect des statuts; il dit y avoir compulsé des ouvrages d'astrologie arabe, le De nativitatibus d'Albubater et le Livre des neuf juges ${ }^{29}$, ce qui laisse à penser qu'il se trouvait alors plutôt dans la "libraria artistarum ». Les boursiers et autres occupants du collège doivent apporter aux livres le plus grand soin : il est interdit d'y porter ses propres annotations, qu'elles soient « interlinearia sive glosas, in marginibus vel in textu ${ }^{30}$, sans avoir auparavant obtenu l'assentiment de la communauté. Un inventaire des livres des deux bibliothèques doit être régulièrement réalisé ; enfin, lors de sa réception au collège, tout nouveau membre doit prêter un serment spécifique, concernant les livres de la bibliothèque et justement intitulé « juramentum pro libraria ». Ce serment mentionne bien l'enchaînement des ouvrages sur les pupitres, ainsi que l'existence d'ouvrages non seulement dans les deux bibliothèques mais aussi dans la chapelle du collège ; peut-être, comme au collège $\mathrm{du}$ Trésorier, est-ce donc dans la chapelle que sont conservés les livres empruntables, quoiqu'il ne soit pas fait mention de bibliothèque de prêt dans les sources. Le fonds de la bibliothèque est régulièrement enrichi par les dons de bienfaiteurs, parfois anciens boursiers du collège.

C'est à compter du $13^{\text {e }}$ siècle que les collèges de l'Université de Paris ont commencé à se doter d'une bibliothèque d'étude, dans le but de contribuer à la formation d'excellents théologiens et prédicateurs séculiers. La plus ancienne et la plus importante de ces bibliothèques, celle du collège de Sorbonne, a su s'inspirer des modèles offerts par les ordres monastiques et mendiants et se les approprier ; les principes de sa réorganisation à la fin du $13^{\text {e }}$ siècle en deux fonds distincts, l'un pour le libre accès, l'autre pour le prêt, ont connu un grand succès et été appliqués dans la plupart des bibliothèques d'étude. On le voit ainsi au collège $\mathrm{du}$ Trésorier, de fondation presque contemporaine, dont l'organisation de la bibliothèque est calquée sur celle du collège de Sorbonne. À l'époque de la fondation du collège de Maître-Gervais, en 1371, il est devenu courant pour ce type d'établissement de disposer de sa propre bibliothèque ; aussi les statuts entrent-ils dans le détail du fonctionnement de cette dernière.

Le registre de prêt de la bibliothèque de Sorbonne au $15^{\mathrm{e}}$ siècle nous laisse entrevoir la richesse de ces fonds et leur rayonnement : nombreux sont en effet les emprunteurs extérieurs au collège ${ }^{31}$. Jusqu'à l'époque moderne, ce sont donc les bibliothèques de collèges et d'institutions régulières qui permirent aux étudiants parisiens d'accéder, à moindres frais, aux ouvrages nécessaires à leurs études.

\section{NOTES}

1- Pierre Riché, Jacques Verger, Des nains sur des épaules de géants : maîtres et élèves au Moyen Âge, Paris, Tallandier, 2006 ; les étudiants devaient entre autres se procurer le vêtement statutaire, le papier nécessaire à la prise de notes, un certain nombre de livres, et s'acquitter du paiement des droits universitaires.

2- Marie-Henriette Jullien de Pommerol, « Livres d'étudiants, bibliothèques de collèges et d'universités ", in Histoire des bibliothèques françaises, tome 1, Paris, Promodis, 1989, p. 93-111

3- Kenneth William Humphreys, «Les bibliothèques des ordres mendiants ", in Histoire des bibliothèques françaises, op. cit., p. 125-145, à la p. 126

4- Claire Angotti, « Les bibliothèques des couvents mendiants, un modèle pour les séculiers ? L'exemple de deux premiers bienfaiteurs de la bibliothèque du collège de Sorbonne (Robert de Sorbon, Gérard d'Abbeville) ", in Entre stabilité et itinérance : livres et culture des ordres mendiants, XIII-XVe siècle, Turnhout, Brepols, 2014, p. 31-72, à la p. 34

5- Claire Angotti, «Bonum commune divinius est quam bonum unius. Le collège de la Sorbonne et sa bibliothèque, place et rôle dans l'Université de Paris au XIV ${ }^{\mathrm{e}}$ siècle ", in Les collèges universitaires en Europe au Moyen Âge et à la Renaissance, Bochum, Winkler, 2011, p. 91-105, à la p. 91 
6- Claire Angotti, « Les bibliothèques des couvents mendiants... », op. cit., p. 32

7- Claire Angotti, art. cit., p. 39

8- Richard H. Rouse, «L'évolution des attitudes envers l'autorité écrite : le développement des instruments de travail au XIII siècle ", in Culture et travail intellectuel dans l'Occident médiéval, Paris, 1981, p. $115-144$

9- Art. cit., p. 122

10- Donatella Nebbiai-Dalla Guarda, « La bibliothèque commune des institutions religieuses ", in Scriptorium, 50, p. 254-268, à la p. 258

11- Donatella Nebbiai-Dalla Guarda, "La bibliothèque commune... ", art. cit., p. 260 ; Kenneth William Humphreys, « Les bibliothèques des ordres mendiants ", art. cit., p. 126.

12- Les sources, notamment un fragment de catalogue de 1275 , semblent indiquer que jusque-là les ouvrages étaient rangés dans des coffres, par matières, et confiés aux bons soins des étudiants (Richard H. et Mary A. Rouse, « La bibliothèque du collège de Sorbonne ", in Histoire des bibliothèques françaises, op. cit., p. 113-123, à la p. 115-116).

13- Art. cit., p. 261

14- Les demandes de Gérard d'Abbeville portent sur : l'établissement d'un procureur auquel sera dévolue la gestion de la bibliothèque ; l'élaboration d'un inventaire, régulièrement mis à jour par le procureur ; l'emprunt possible des ouvrages contre caution ; la conservation des ouvrages (Claire Angotti, «Les bibliothèques des couvents mendiants », op. cit., p. 47). Le fragment d'inventaire réalisé en 1275, soit peu après le legs de Gérard d'Abbeville, semble suggérer que les dispositions qu'il indique dans son testament ont été respectées (Richard H. et Mary A. Rouse, « La bibliothèque du collège de Sorbonne », op. cit., p. 116). On retrouve par la suite la plupart de ces dispositions dans les statuts réglant le fonctionnement des bibliothèques de collèges.

15- Les copies du texte du testament, en l'absence de l'original, donnent deux lectures différentes de cette phrase. Claire Angotti («Les bibliothèques des couvents mendiants », op. cit., p. 47) penche néanmoins pour la leçon demandant l'enchaînement des usuels.

16- Les statuts, du 18 août 1280, sont connus par des copies, dont celle du ms 102 de la bibliothèque de la Sorbonne ; concernant la bibliothèque, ils mentionnent l'interdiction de faire sortir des ouvrages du collège, et la nécessité de veiller à leur bonne conservation («Item, nolumus quod libri accommodentur per villam ad transcribendum, vel etiam scribendum, quia sic possent diminui, vel etiam inquinari ; et volumus quod eorum custodia iis deputetur qui valeant de illis respondere integre, et qui aliis ministrent et dividant, prout viderint expedire »).

17- Il est conservé aux Archives nationales sous la cote AN M $194 \mathrm{n}^{\circ} 5$.

18- Sur les bibliothèques des petits collèges parisiens, et entre autres celles du collège du Trésorier, voir Karine Klein, Les livres des petits collèges à Paris aux XIV et $X V^{e}$ siècles, thèse de doctorat en histoire, sous la direction de Jean-Philippe Genet, Université Paris I, 2005.

19- Karine Klein, op. cit., p. 177

20- Op. cit., p. 209

21- AN M $163 \mathrm{n}^{\circ} 19$

22- AN M $163 \mathrm{n}^{\circ} 19$, chapitre huit

23 - Ibid.
$24-$ Ibid.

25- «Et ideo racionabile, congruum et utile est quod ligentur cum cathenis sicut in aliis librariis dicti collegii, ut ibi fermius conserventur, et ne quod multis debet proficere per unius negligenciam vel deffectum deperdatur, et in hoc intencio et voluntas fundatoris defraudetur " (AN, M $\left.164 \mathrm{n}^{\circ} 6\right)$.

26- AN M $167 \mathrm{n}^{\circ} 19$

27- Sur ce point, le collège de Sorbonne fait exception : sa bibliothèque est ouverte à tous les membres de l'université.

28- Ces recommandations à l'égard des personnes extérieures à l'établissement sont assez habituelles dans les règlements des bibliothèques de collèges (Karine Klein, op. cit., p. 215).

29- À propos de l'ouvrage intitulé De nativitatibus d'Albubater, Simon de Phares écrit : « lequel livre ay veu au Colliege du roy Charles le Quint, en la librairie de astrologie a Paris jouxte le Livre des IX Juges, bien correct et approuvé et, par ce, incathené et en usage a qui en veult " (Jean-Patrice Boudet, éd., Le Recueil des plus célèbres astrologues de Simon de Phares, Paris, 1994, tome I, p. 293). Si aucun des ouvrages actuellement conservés et issus de la bibliothèque du collège ne correspond aux deux titres cités, le témoignage de l'astrologue n'en est pas moins crédible.

30- AN M $163 n^{\circ} 19$, chapitre huit

31- Jeanne Vielliard et Marie-Henriette Jullien de Pommerol (éd.), Le registre de prêt de la bibliothèque du collège de Sorbonne, 1402-1536, Paris, CNRS éditions, 2000 